\title{
Editorial: Quantitative Analysis of Neuroanatomy
}

\author{
Julian M. L. Budd ${ }^{1 *}$, Hermann Cuntz ${ }^{2,3}$, Stephen J. Eglen ${ }^{4}$ and Patrik Krieger ${ }^{5}$ \\ ${ }^{1}$ Department of Informatics, School of Engineering and Informatics, University of Sussex, Brighton, UK, ${ }^{2}$ Ernst Strüngmann \\ Institute for Neuroscience in Cooperation with Max Planck Society, Frankfurt/Main, Germany, ${ }^{3}$ Frankfurt Institute for \\ Advanced Studies, Frankfurt/Main, Germany, ${ }^{4}$ Department of Applied Mathematics and Theoretical Physics, Cambridge \\ Computational Biology Institute, University of Cambridge, Cambridge, UK, ${ }^{5}$ Department of Systems Neuroscience, Medical \\ Faculty, Ruhr University Bochum, Bochum, Germany
}

Keywords: neuroinformatics, connectome, mathematical modeling, statistical analysis, quantitative methods, computational neuroscience

\section{INTRODUCTION}

The true revolution in the age of digital neuroanatomy is the ability to extensively quantify anatomical structures and thus investigate structure-function relationships in great detail. Largescale projects were recently launched with the aim of providing infrastructure for brain simulations. These projects will increase the need for a precise understanding of brain structure, e.g., through statistical analysis and models.

From articles in this Research Topic, we identify three main themes that clearly illustrate how new quantitative approaches are helping advance our understanding of neural structure and function. First, new approaches to reconstruct neurons and circuits from empirical data are aiding neuroanatomical mapping. Second, methods are introduced to improve understanding of the underlying principles of organization. Third, by combining existing knowledge from lower levels of organization, models can be used to make testable predictions about a higher-level organization where knowledge is absent or poor. This latter approach is useful for examining statistical properties of specific network connectivity when current experimental methods have not yet been able to fully reconstruct whole circuits of more than a few hundred neurons.

\section{OPEN ACCESS}

Edited by: Javier DeFelipe, Cajal Institute, Spain

*Correspondence: Julian M. L. Budd j.m.l.budd@sussex.ac.uk

Received: 13 October 2015 Accepted: 26 October 2015 Published: 11 November 2015

Citation:

Budd JML, Cuntz H, Eglen SJ and Krieger P (2015) Editorial: Quantitative Analysis of Neuroanatomy.

Front. Neuroanat. 9:143. doi: 10.3389/fnana.2015.00143

\section{RECONSTRUCTION}

The first theme illustrates how novel quantitative anatomical methods are reducing the time and effort taken to reconstruct neurons and networks even when data are incomplete.

Modeling the electrophysiological computations made by single neurons requires a precise reconstruction of their morphology. Blackman et al. (2014) assessed the accuracy of neuronal reconstructions of biocytin-labeled cells against reconstructions from fluorescence-based imaging using 2-photon microscopy. The authors conclude that biocytin-labeled cells are more accurate at reproducing diameter values which is in particular crucial for electrophysiology modeling, while faster fluorescence-imaging reconstruction methods are appropriate for tasks such as cell-type classification.

Identifying cell types based on accurate tracings is very time-consuming. From such tracings of microscopic images, it is known that retinal cell types can be identified by their laminar position in the network (Sümbül et al., 2014a). Taking advantage of this link between macroscopic and microscopic features, Sümbül et al. (2014b) show how automated volumetric reconstructions can be performed more rapidly using the fluorescence distribution directly obtained from the image stacks. 
Integrating structural and functional data has always been central to reconstructing neural circuits. Here, Ullo et al. (2014) apply a novel method that uses structural data of in vitro neuronal networks to constrain estimates of functional connections underlying spiking data of the same network acquired with microelectrode arrays. The general idea could also apply to the more complex structures in vivo.

Our understanding of synaptic connectivity is largely based on measurements from brain slice preparations. However, some of the complex 3D geometry of neurons is unavoidably lost by slicing. This problem affects connectivity measurements, especially for long-range connections. Two articles address this problem. First, van Pelt et al. (2014) validate a statistical approach (implemented in the NETMORPH software) for inferring complete neuronal reconstructions from incomplete slice data. From these completed neuronal morphologies, the authors explain how this information can be used to estimate connectivity in large-scale networks. Second, Miner and Triesch (2014) use a computational model to propose how differences in the experimental procedure such as slice thickness and sampling area can explain differences observed in experimentally-derived results.

Abnormalities in subcellular organelle morphology and distribution characterize a variety of neuropathological conditions. To aid faster quantification, Perez et al. (2014) combine image processing methods with a supervised, multiresolution machine learning algorithm to automatically segment specific types of cellular organelles (mitochondria, lysosomes, nuclei, and nucleoli) from electron microscopy (EM) image stacks. The authors demonstrate how this approach should generalize to other organelle types and scale to large 3D datasets from serial electron microscopy.

\section{DESCRIPTION}

The second theme in this Research Topic is that mathematical techniques are applied to describe the spatial properties of neurons and networks at a range of scales (Eglen et al., 2008; Hansson et al., 2013). Firstly, two papers used spatial statistics to examine spatial patterning within a region of neural tissue. Anton-Sanchez et al. (2014) studied the spatial distribution of synapses in layers I to VI of rat cortex in three dimensions. They found that synapses are distributed randomly, subject only to not physically overlapping with each other, although density variations were found between different layers. Moving from the distribution of synapses to distributions of neurons, Keeley and Reese (2014) proposed a new metric for evaluating the spatial regularity in two-dimensional distributions of neuronal somata. They suggest a normalization term for the widely-used regularity index measure that compares the average and the standard deviation of the distance between cells. Using various genetically inbred strains their new measure is found to be effective in detecting genes controlling spatial patterning in various genetically inbred strains using quantitative trait loci approaches.

The papers by Anton-Sanchez et al. (2014) and Keeley and Reese (2014) both analyse the distribution of synapses and neurons by treating these objects as points in space. Polavaram et al. (2014) by contrast studied the detailed morphology of individual neurons to search for key features underlying the variability in axonal and dendritic morphologies. By comparing around 5000 neuron morphologies curated from the Neuromorpho.org repository, they discovered six main morphological classes, with clustering driven mainly by biological factors, such as cell type, rather than technical factors, such as recording laboratory.

The study by Polavaram et al. (2014) highlights the difficulties in performing quantitative analysis of data recorded across many laboratories. Instead of reanalysing raw data, Beul and Hilgetag (2014) performed a detailed literature review of rodent cortex anatomy to evaluate the evidence for a universal "canonical microcircuit" (Douglas and Martin, 2004). Beul and Hilgetag suggest such a canonical microcircuit, proposed based on data from cat striate cortex, is unlikely to apply into other regions of the cortex where the granular layer (layer 4) is reduced or absent-the "agranular areas" of cortex. Instead, they propose a revised wiring diagram, with reduced inhibition between upper and deep layers in these agranular regions.

\section{GENERATIVE MODELING}

The third and final theme that emerged from this collection of articles concerns the usage of generative methods to bridge the gap between single neurons and the overall network structure (Budd and Kisvárday, 2012). Computational models sometimes based on simple self-organizing principles exist that reproduce biology at a high level of detail both at the microscopic and macroscopic scales (Schneider et al., 2014). Complementing these approaches, large databases have emerged that embed the biological details captured at the microscopic scale into the context of larger scale structures (Chiang et al., 2011). The resulting generative approaches allow for a better intuition of the underlying principles for higher-level organization and for making predictions in cases where data are currently sparse or missing.

Egger and colleagues provide a software package NeuroNet that generates a statistical connectome model of the barrel cortex while taking into account statistical measures of synapse and soma distributions as well as a small subset of complete realistic cellular morphologies for all cell types (Egger et al., 2014). The resulting statistical connectome is in line at all scales with experimental anatomical and electrophysiological measurements in barrel cortex and indicates that cortical connections are probabilistic as a function of dendrite and axon overlap.

In the study by Egger et al. registering the reconstructed morphologies and synaptic positions to the barrel outlines is essential to generate their statistical connectome. In a similar line, the context in the circuit is the defining feature of the study by Torben-Nielsen and de Schutter that provides a software tool in Python called NeuroMaC to generate synthetic dendritic morphologies within the constraints provided by a given piece of tissue (Torben-Nielsen and De Schutter, 2014). The synthetic neuronal morphologies are grown both considering the context 
of large-scale circuit features and the interactions with other growing dendritic trees in the vicinity.

Aćimović and colleagues study the relationship between dendritic shape and network measures using statistical models at both micro- and macroscopic scales albeit in a simplified setting that allows analytical solutions to be obtained (Acimovic et al., 2015). For analytical tractability, the small scale dendritic and axonal shapes are described by density fields that predict network motif distributions and can be compared with experimental data (Song et al., 2005; Rieubland et al., 2014).

Brain disorders are often accompanied by alterations in the higher level context in which neurons are embedded. Using a model of structural plasticity, Butz et al. (2014) analyse how such global structural changes can affect network connectivity. It is argued that local homeostatic structural plasticity mechanisms can cause changes in network topology.

With the parametric anatomical modeling (PAM) technique by Pyka et al. (2014) the precise shape of macroscale anatomical structures is incorporated into neural network models. Using empirically-based mapping rules and connectivity kernels to generate realistic pathway trajectories, spatial connectivity matrices, and axonal conduction distances, Pyka and colleagues make testable predictions for interlaminar connectivity parameter distributions.

Finally, long-range connections require fast and reliable axonal signal propagation and Neishabouri and Faisal (2014) have studied a recently observed structural formation of proteins and lipids known as lipid rafts (Pristerà et al., 2012) in thin, unmyelinated axons in the peripheral nervous

\section{REFERENCES}

Acimovic, J., Mäki-Marttunen, T., and Linne, M.-L. (2015). The effects of neuron morphology on graph theoretic measures of network connectivity: the analysis of a two-level statistical model. Front. Neuroanat. 9:76. doi: 10.3389/fnana.2015.00076

Anton-Sanchez, L., Bielza, C., Merchán-Pérez, A., Rodríguez, J.-R., DeFelipe, J., and Larrañaga, P. (2014). Three-dimensional distribution of cortical synapses: a replicated point pattern-based analysis. Front. Neuroanat. 8:85. doi: 10.3389/fnana.2014.00085

Beul, S. F., and Hilgetag, C. C. (2014). Towards a "canonical" agranular cortical microcircuit. Front. Neuroanat. 8:165. doi: 10.3389/fnana.2014.00165

Blackman, A. V., Grabuschnig, S., Legenstein, R., and Sjöström, P. J. (2014). A comparison of manual neuronal reconstruction from biocytin histology or 2photon imaging: morphometry and computer modeling. Front. Neuroanat. 8:65. doi: 10.3389/fnana.2014.00065

Budd, J. M. L., and Kisvárday, Z. F. (2012). Communication and wiring in the cortical connectome. Front. Neuroanat. 6:42. doi: 10.3389/fnana.2012.00042

Butz, M., Steenbuck, I. D., and van Ooyen, A. (2014). Homeostatic structural plasticity can account for topology changes following deafferentation and focal stroke. Front. Neuroanat. 8:115. doi: 10.3389/fnana.2014.00115

Chiang, A.-S., Lin, C.-Y., Chuang, C.-C., Chang, H.-M., Hsieh, C.-H., Yeh, C.W., et al. (2011). Three-dimensional reconstruction of brain-wide wiring networks in Drosophila at single-cell resolution. Curr. Biol. 21, 1-11. doi: 10.1016/j.cub.2010.11.056

Douglas, R. J., and Martin, K. A. C. (2004). Neuronal circuits of the neocortex. Annu. Rev. Neurosci. 27, 419-451. doi: 10.1146/annurev.neuro.27.070203. 144152

Egger, R., Dercksen, V. J., Udvary, D., Hege, H.-C., and Oberlaender, M. (2014). Generation of dense statistical connectomes from sparse morphological data. Front. Neuroanat. 8:129. doi: 10.3389/fnana.2014.00129 system. Using realistic stochastic modeling of individual ion channels, Neishabouri and Faisal show that while action potential conduction in such systems was reliable, it did not offer any obvious gain in either conduction velocity or metabolic cost over a uniform ion channel density.

\section{CONCLUSION}

With these articles we hope the reader will appreciate that understanding neural structure quantitatively and its functional relations is more than a handle turning exercise of known algorithms but a creative interdisciplinary endeavor of a variety of approaches across different species, brain regions, and spatial scales. Here, authors have managed to coax information out of noisy data obtained at the extremes of methodological resolution; they have discovered new ways of describing anatomical organization; and arrived at novel ideas that when implemented in a generative way provide an anatomical framework for large-scale network models of the brain. To maximize this creativity from limited funding, there is an obvious need to provide an environment in which individual exploration, data access, collaboration, and reproducibility is made much easier through an open and shared informatics framework (Green et al., 2015).

\section{ACKNOWLEDGMENTS}

HC was funded by the German Federal Ministry of Education and Research grant 01GQ1406.

Eglen, S. J., Lofgreen, D. D., Raven, M. A., and Reese, B. E. (2008). Analysis of spatial relationships in three dimensions: tools for the study of nerve cell patterning. BMC Neurosci. 9:68. doi: 10.1186/1471-2202-9-68

Green, E. D., Watson, J. D., and Collins, F. S. (2015). Human genome project: twenty-five years of big biology. Nature 526, 29-31. doi: 10.1038/526029a

Hansson, K., Jafari-Mamaghani, M., and Krieger, P. (2013). RipleyGUI: software for analyzing spatial patterns in 3D cell distributions. Front. Neuroinform. 7:5. doi: 10.3389/fninf.2013.00005

Keeley, P. W., and Reese, B. E. (2014). The patterning of retinal horizontal cells: normalizing the regularity index enhances the detection of genomic linkage. Front. Neuroanat. 8:113. doi: 10.3389/fnana.2014.00113

Miner, D. C., and Triesch, J. (2014). Slicing, sampling, and distance-dependent effects affect network measures in simulated cortical circuit structures. Front. Neuroanat. 8:125. doi: 10.3389/fnana.2014.00125

Neishabouri, A., and Faisal, A. A. (2014). Saltatory conduction in unmyelinated axons: clustering of $\mathrm{Na}+$ channels on lipid rafts enables micro-saltatory conduction in C-fibers. Front. Neuroanat. 8:109. doi: 10.3389/fnana.2014. 00109

Perez, A. J., Seyedhosseini, M., Deerinck, T. J., Bushong, E. A., Panda, S., Tasdizen, T., et al. (2014). A workflow for the automatic segmentation of organelles in electron microscopy image stacks. Front. Neuroanat. 8:126. doi: 10.3389/fnana.2014.00126

Polavaram, S., Gillette, T. A., Parekh, R., and Ascoli, G. A. (2014). Statistical analysis and data mining of digital reconstructions of dendritic morphologies. Front. Neuroanat. 8:138. doi: 10.3389/fnana.2014.00138

Pristerà, A., Baker, M. D., and Okuse, K. (2012). Association between tetrodotoxin resistant channels and lipid rafts regulates sensory neuron excitability. PLoS ONE 7:e40079. doi: 10.1371/journal.pone.0040079

Pyka, M., Klatt, S., and Cheng, S. (2014). Parametric anatomical modeling: a method for modeling the anatomical layout of neurons and their projections. Front. Neuroanat. 8:91. doi: 10.3389/fnana.2014.00091 
Rieubland, S., Roth, A., and Häusser, M. (2014). Structured connectivity in cerebellar inhibitory networks. Neuron 81, 913-929. doi: 10.1016/j.neuron. 2013.12.029

Schneider, C. J., Cuntz, H., and Soltesz, I. (2014). Linking macroscopic with microscopic neuroanatomy using synthetic neuronal populations. PLoS Comput. Biol. 10:e1003921. doi: 10.1371/journal.pcbi.1003921

Song, S., Sjöström, P. J., Reigl, M., Nelson, S., and Chklovskii, D. B. (2005). Highly nonrandom features of synaptic connectivity in local cortical circuits. PLoS Biol. 3:e68. doi: 10.1371/journal.pbio.0030068

Sümbül, U., Song, S., McCulloch, K., Becker, M., Lin, B., Sanes, J. R., et al. (2014a). A genetic and computational approach to structurally classify neuronal types. Nat. Commun. 5, 3512. doi: 10.1038/ncomms4512

Sümbül, U., Zlateski, A., Vishwanathan, A., Masland, R. H., and Seung, H. S. (2014b). Automated computation of arbor densities: a step toward identifying neuronal cell types. Front. Neuroanat. 8:139. doi: 10.3389/fnana.2014.00139

Torben-Nielsen, B., and De Schutter, E. (2014). Context-aware modeling of neuronal morphologies. Front. Neuroanat. 8:92. doi: 10.3389/fnana.2014. 00092
Ullo, S., Nieus, T. R., Sona, D., Maccione, A., Berdondini, L., and Murino, V. (2014). Functional connectivity estimation over large networks at cellular resolution based on electrophysiological recordings and structural prior. Front. Neuroanat. 8:137. doi: 10.3389/fnana.2014.00137

van Pelt, J., van Ooyen, A., and Uylings, H. B. M. (2014). Axonal and dendritic density field estimation from incomplete single-slice neuronal reconstructions. Front. Neuroanat. 8:54. doi: 10.3389/fnana.2014.00054

Conflict of Interest Statement: The authors declare that the research was conducted in the absence of any commercial or financial relationships that could be construed as a potential conflict of interest.

Copyright (c) 2015 Budd, Cuntz, Eglen and Krieger. This is an open-access article distributed under the terms of the Creative Commons Attribution License (CC BY). The use, distribution or reproduction in other forums is permitted, provided the original author(s) or licensor are credited and that the original publication in this journal is cited, in accordance with accepted academic practice. No use, distribution or reproduction is permitted which does not comply with these terms. 